\title{
ADHD and stimulant drug treatment: what can the children teach us?
}

The treatment of children diagnosed with attention deficit/hyperactivity disorder (ADHD) with stimulant drugs has been a subject of controversy for many years, both within and outside bioethics, and the controversy is still very much alive. In her feature article (see page 359, Editor's choice), Ilina Singh, a major contributor to that debate in recent years, brings fresh empirical evidence to bear on it. She uses new data to deal with two key ethical concerns that have been raised about the practice. First, does medicating children with ADHD compromise their capacity for autonomous moral agency? And second, does it pose a threat to their 'authentic self'? A related question is whether medication for ADHD is being used as an instrument of social control, forcing children to adapt to environments that they find oppressive.

Whereas previous research by Singh examined the attitudes of parents of boys diagnosed with ADHD, ${ }^{1}$ this article draws from a series of interviews with children (more than 150) from the UK and USA, including those who were taking drugs for ADHD. The conclusions that Singh reaches can be characterised as cautiously optimistic. The data, she thinks, indicate that 'a majority of children are not victims of stimulant drugs'. Rather than experiencing their use of such drugs as undermining their capacity for moral agency, children tend to report that the medication renders them better able to control their responses in potentially challenging situations. In addition, most of the children did not perceive the medication as a threat to their authenticity. Singh nevertheless accepts that such a threat might be real in certain cases, and suggests ways in which the threat might be mitigated. In particular, she suggests-plausibly, I think-that medical professionals could play a key role by spending more time listening to children in order to get a deeper understanding of the individual nature of their problems, rather than relying on a more cursory approach that is limited to diagnosing and treating the symptoms of ADHD.

Correspondence to Mr Alexandre Erler, Oxford Uehiro Centre for Practical Ethics, University of Oxford, Suite 8, Littlegate House, 16/17 St Ebbe's Street, Oxford OX1 1PT, UK; alexandre.erler@gmail.com
In this concise argument, I wish to take a brief look at the two main ethical issues discussed by Singh. The concern about moral agency is two-pronged. First, one might worry that reliance on stimulant drugs might lead the children who use them to view themselves as helpless slaves of their neurochemistry, needing a pill to make them act rightly, rather than as autonomous moral agents. Second, one might fear that a dependence on medication will prevent these children from developing a fully-fledged capacity for self-control and moral agency. The evidence presented by Singh does, I believe, successfully alleviate the former worry, and even suggests that it has got things back to front: children interviewed typically insist that medication for ADHD increases their ability to choose freely how to act (in the words of one interviewee, it does not turn the user into a 'robot'), and it seems that many of them would not feel that they were in the driver's seat' without such medication.

Singh's data also go some way towards alleviating the second worry about medication having a detrimental effect on the development of moral agency in children. Indeed, both the children's self-reports and independent behavioural evidence suggest that ADHD, at least in its florid forms, does impair one's capacity for selfcontrol and autonomous decision-making. As Art Caplan notes in his commentary (see page 367), children seem more likely to successfully develop such capacities if they do not need to constantly grapple with a tendency to get distracted or act impulsively. In some cases, developing capacities for self-control and autonomous decision-making might be impossible without medication, whereas in others they might be attainable yet still much more difficult than normal to achieve; should we really want things to be as hard as possible for children who fall into the latter category, when we have clear ways of helping them? I will just add one qualification here. The worry about psychostimulants becoming substitutes for true character-building may, it seems to me, have more plausibility on a prior assumption of misdiagnosis. Although we can agree with Caplan that we all sometimes need a helping hand to manage to act as we should, it would still seem problematic if children showing features reminiscent of ADHD, but with no significant impairment to their capacity for self-governance, were mistakenly led to believe that they did have such an impairment, and that they should seek pharmacological help to overcome temptations and challenges that they might reasonably be expected to manage on their own. This worry raises the difficult question of the frequency with which ADHD is being misdiagnosed in children.

As Singh notes, the concern about authenticity can also take two forms, depending on whether our understanding of this concept emphasises self-discovery or self-creation. ${ }^{2}$ A supporter of the former approach might worry that medication for ADHD stifles valuable aspects of a child's 'authentic self', such as creativity or spontaneity. On the self-creation approach, the concern will relate to the possibility that the values and goals promoted by stimulant drug treatment are not really the child's 'own'-but rather, say, those of his/her parents. Both approaches, we may note, distinguish between authenticity proper and children's subjective perception of authenticity (whether their own, or that of their peers). The data discussed by Singh show that the latter is sometimes adversely affected by medication, from which one might infer that the actual authenticity of the child is threatened as well: some of her interviewees reported no longer feeling like themselves and having become less 'fun to be around' in their friends' eyes while receiving medication. That said, Singh mentions that the number of such cases is small. Moreover, as Steven Hyman points out (see page 369), the effects of psychostimulants are transient: children will return to their typical baseline by the end of the school day unless given an additional dose, and many of them stop their medication completely at week-ends and during school holidays. This suggests that even if there are cases where the medication stifles a valuable part of the child's authentic self, this sacrifice is usually temporary, confined to periods during which it would lead to maladaptive behaviour. And one might find such a tradeoff acceptable, at least when no adequate alternative to pharmacological treatment is available. No matter 
how valuable authenticity (in the selfdiscovery sense) might be, it is not clear that it must always trump other considerations, including the prudential interest of the child in completing compulsory education. The question of authenticity in this sense is complicated by the possibility, alluded to by Singh, that by suppressing one aspect of the child's authentic self, the medication might nevertheless allow another one (say, a taste for some particular academic subject), which had been overshadowed by the previous one, to finally be expressed-an interesting thought which deserves further consideration and discussion.

Concerns about authenticity in the selfcreation sense, which can also be expressed in terms of concerns about autonomy, are raised by Steven Rose (see page 371). Although the children interviewed by Singh do report valuing the increased capacity to meet social expectations that stimulants provide, this is not enough to quiet the above concerns, as theorists of autonomy usually place further constraints on the sort of evaluative attitudes that can count as autonomous (eg, that they would survive a process of critical reflection), making autonomy difficult to assess in cases such as those discussed here. Rose also calls into question the conclusions drawn by Singh from these interviews, suggesting that the children might merely be repeating what they were told by their parents and educators, with their discourse concealing the fact that the symptoms for which they are being treated might represent a legitimate response to oppressive circumstances and expectations. I will let readers assess the persuasiveness of Rose's critique for themselves, yet would like to raise the following general question in response: If we cannot trust the children's reports because they can only parrot the views of their parents and teachers, and if we cannot listen to parents and teachers because of their tendency to misdescribe the situation in accordance with their own prejudices, whose observations (as opposed to mere speculations) are we to rely on to develop an accurate picture? Radically critical views of stimulant drug treatment seem to rest chiefly, as Singh suggests in her response (see page 372), on general a priori claims about children with ADHD. Proponents of such views typically do not provide any concrete evidence demonstrating that these children's personal circumstances can be adequately described as oppressive, or that environmental interventions (say, a different learning structure), unsupplemented by medication, would allow them-all of them, or at least the vast majority of them-to function successfully. It seems to me that this places such views on shaky ground.

The ethical debate on the pharmacological treatment of children diagnosed with ADHD will, and needs to, continue. Several concerns, such as that of misdiagnosis, still need to be tackled more effectively. However, I believe that Singh's latest contribution, and the responses to it, together make a strong case for the value of an empirically grounded approach, and the need to move towards more nuanced views which recognise both the benefits and potential ethical pitfalls of stimulant drug treatment for ADHD in childrenrather than unqualifiedly embracing or condemning the practice.

Competing interests None.

Provenance and peer review Commissioned internally peer reviewed.

\section{REFERENCES}

1 Singh I. Will the "real boy" please behave: dosing dilemmas for parents of boys with ADHD. Am J Bioethics 2005;5(3):34-47.

2 Following Neil Levy's distinction in Levy N. Enhancing authenticity. J App/ Philos 2011;28(3):308-18. 